\title{
Le coût attentionnel associé aux fonctions exécutives impliquées dans le contrôle postural
}

\author{
M. Boisgontier^, J.-B. Mignardot, V. Nougier, I. Olivier et E. Palluel \\ UJF-Grenoble 1 / CNRS / TIMC-IMAG UMR 5525, Grenoble, F-38041, France
}

Reçu le 30 mars 2011 - Accepté le 12 mai 2011

\begin{abstract}
Résumé. Le présent article s'efforce de faire une synthèse des différents travaux liés au coût attentionnel du contrôle postural. Dans un contexte de double tâche, la mobilisation des ressources attentionnelles associées au contrôle de la posture dépend de plusieurs facteurs tels que (1) la focalisation attentionnelle volontaire ou automatique sur le contrôle postural, (2) les informations sensorielles disponibles, (3) la nature et la complexité de la tâche posturale, (4) l'âge, ou encore (5) le niveau d'expertise ou de handicap des sujets. Après une rapide présentation du paradigme de la double tâche dans le cadre du contrôle postural, les effets de ces différents facteurs sont analysés plus spécifiquement.
\end{abstract}

Mots clés : Posture, attention, double tâche, âge, pathologie

\begin{abstract}
Attentional cost of the executive functions involved in postural control.
The present paper tried to provide a review of the different works investigating the attentional cost of postural control. Within the context of a dual-task, the attentional demand associated with postural control depends on various factors such as (1) the automatic or voluntary focalisation of attention on postural control, (2) the available sensory information, (3) the nature and complexity of the postural task, (4) the subjects' age, and/or (5) the level of subjects' sensory-motor expertise or deficits. Following a brief presentation of the dual-task paradigm in the context of postural control, the effects of these different factors are analysed more specifically.
\end{abstract}

Key words: Posture, attention, dual-task, age, pathology

\section{Introduction}

Pendant longtemps, le contrôle postural a été considéré comme une réponse automatique à une perturbation donnée (Belenkii, Gurfinkel, \& Paltsev, 1967). Cependant, des données récentes ont mis en évidence le fait que la cognition et le contrôle postural s'appuient sur des mécanismes communs, ce qui engendre souvent des relations conflictuelles (Kerr, Condon, \& McDonald, 1985; Massion, 1992; Woollacott \& Shumway-Cook, 2002), et remet en cause l'hypothèse selon laquelle le contrôle postural dépend uniquement de processus spinaux ou subcorticaux. En d'autres termes, la régulation de la posture mobilise des ressources attentionnelles ou cognitives (Vuillerme \& Nougier, 2004; Woollacott \& ShumwayCook, 2002).

\footnotetext{
* Les auteurs sont présentés par ordre alphabétique, la contribution de chacun étant équivalente.
}

L'interaction entre processus cognitifs et contrôle postural est typiquement étudiée au travers du paradigme dit de la double tâche (Abernethy, 1988) dans lequel les deux tâches considérées peuvent plus ou moins entrer en compétition (Andersson, Hagman, Talianzadeh, Svedberg, \& Larsen, 2002; Dault, Geurts, Mulder, \& Duysens, 2001; Melzer, Benjuya, \& Kaplanski, 2001; Pellechia, 2003; Shumway-Cook, Woollacott, Kerns, \& Baldwin, 1997). Ce paradigme de la double tâche s'appuie sur un certain nombre de principes méthodologiques qu'il convient de rappeler. Tout d'abord, les sujets sont confrontés à deux tâches données, réalisées séparément, et pour lesquelles on mesure la performance réalisée. Ensuite, les sujets sont invités à réaliser ces deux tâches simultanément. Dans cette condition de double tâche, l'une des deux tâches est définie comme étant la tâche primaire (e.g., maintien postural) que les sujets doivent réaliser de manière prioritaire et si possible avec le même niveau de performance que lorsque la tâche considérée est exécutée 


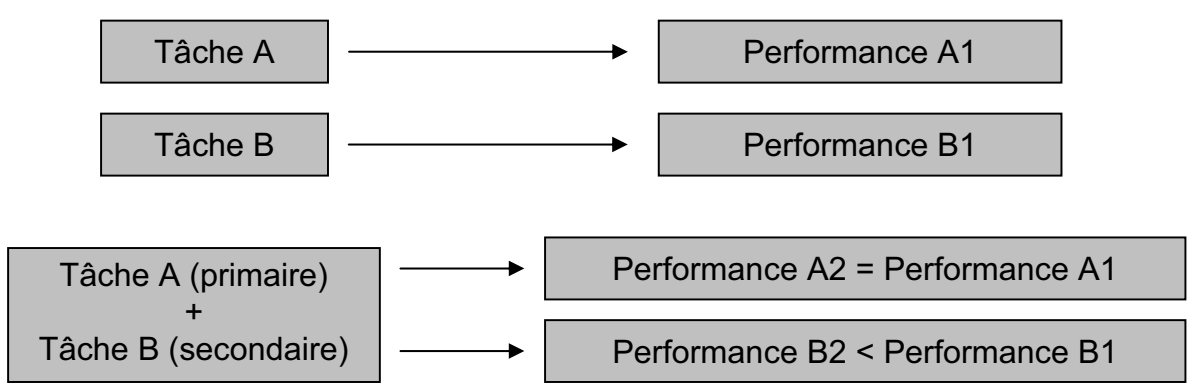

Fig. 1. Illustration schématique du paradigme de la double tâche. Ce paradigme est utilisé pour mesurer le coût attentionnel d'une tâche appelée primaire.

seule. L'autre tâche, concurrente, est appelée tâche secondaire (e.g. tâche cognitive de Stroop ou compte à rebours) et doit être réalisée du mieux possible. Lorsque les capacités attentionnelles du système nerveux central (SNC) sont dépassées, de par la concurrence entre les deux tâches, la performance dans la tâche secondaire diminue. Cette baisse de performance dans la tâche qualifiée de secondaire rend compte du coût attentionnel nécessaire pour réaliser la tâche primaire (Fig. 1).

En d'autres termes, quand la tâche concurrente peut être réalisée sans dépasser les ressources attentionnelles laissées disponibles par la tâche dite primaire, sa performance n'est pas affectée. L'interférence entre deux tâches apparaît seulement lorsque les exigences de ces dernières dépassent les capacités attentionnelles du SNC (Bourke, Duncan, \& Nimmo-Smith, 1996). Yardley et al. (2001) ont montré que cette interférence est principalement à attribuer aux capacités limitées de traitement de l'information d'un individu et qu'elle est proportionnelle à la demande attentionnelle des deux tâches à effectuer. Cette interférence dépend également de la nature de la concurrence mise en jeu : sollicitation ou non des mêmes processus perceptifs, cognitifs, et/ou moteurs.

Outre le paradigme de la double tâche précédemment décrit qui permet de déterminer le coût attentionnel d'une tâche primaire, il est possible d'utiliser une situation de double tâche dans le but de déceler les stratégies de partage de l'attention développées par un individu. Dans ce cas, il ne s'agit pas de lui demander de maintenir le niveau de performance d'une tâche lorsqu'elle est réalisée en même temps qu'une autre, mais au contraire d'identifier la priorité qu'il donne à l'une ou à l'autre des deux tâches. Ainsi, on observe soit une dégradation des deux tâches, soit une dégradation uniquement de l'une ou l'autre des tâches (Fig. 2).

Dans un contexte de double tâche, la mobilisation des ressources attentionnelles associées au contrôle de la posture dépend de plusieurs facteurs tels que (1) la focalisation attentionnelle volontaire ou automatique sur le contrôle postural (Vuillerme \& Nafati, 2007), (2) les informations sensorielles disponibles (Shumway-Cook \& Woollacott, 2000; Teasdale \& Simoneau, 2001), (3) la nature et la complexité de la tâche posturale (Lajoie, Teasdale, Bard, \& Fleury, 1996), (4) l'âge (Teasdale \&
Simoneau, 2001 ; Woollacott \& Shumway-Cook, 2002), ou encore (5) le niveau d'expertise (Vuillerme \& Nougier, 2004) ou de handicap des sujets (Lajoie, Barbeau, \& Hamelin, 1999; Mignardot, Olivier, Promayon, \& Nougier, 2010). Ces différents points sont repris plus spécifiquement dans ce qui suit.

\section{Effets de la focalisation attentionnelle}

Différents travaux ont tenté de déterminer quels étaient les effets d'une focalisation attentionnelle sur la stabilité posturale lorsque l'individu se trouvait en situation de double tâche. Les résultats obtenus sont parfois contradictoires. Certains travaux montrent une dégradation du contrôle postural (Andersson, Yardley, \& Luxon, 1998; Maki \& McIlroy, 1996; Rankin, Woollacott; ShumwayCook, \& Brown, 2000, Shumway-Cook \& Woollacott, 2000), tandis que d'autres font état d'une absence d'effet (Stelmach, Zelaznik, \& Lowe, 1990; Yardley, Gardner, Leadbetter, \& Lavie, 1999) voire même d'une amélioration (Kerr et al., 1985) du contrôle postural en situation de double tâche. Pour tenter d'approfondir cette question, Vuillerme, Nougier, \& Teasdale (2000) ont étudié les effets de tâches de temps de réaction plus ou moins complexes, sur le contrôle postural, avant, durant et après l'exécution de la double tâche. Dans une condition contrôle, aucune double tâche n'était exécutée et les sujets devaient focaliser leur attention sur la stabilisation de leur posture. Les résultats ont clairement montré que la stabilité posturale était meilleure lorsque les sujets focalisaient leur attention sur une autre tâche que le contrôle postural.

De manière similaire, Vuillerme \& Nafati (2007) ont observé une dégradation de la stabilité posturale quand des sujets adultes devaient volontairement focaliser leur attention sur leur stabilité posturale. Une explication possible de cette dégradation est que la focalisation attentionnelle sur la posture implique une augmentation du contrôle volontaire qui vient interférer avec les processus habituels de contrôle automatique généralement sollicités (Wulf \& Prinz, 2001). D’un point de vue neurologique, les dégradations observées lorsque l'attention est volontairement focalisée sur le mouvement (Zachry, Wulf, 


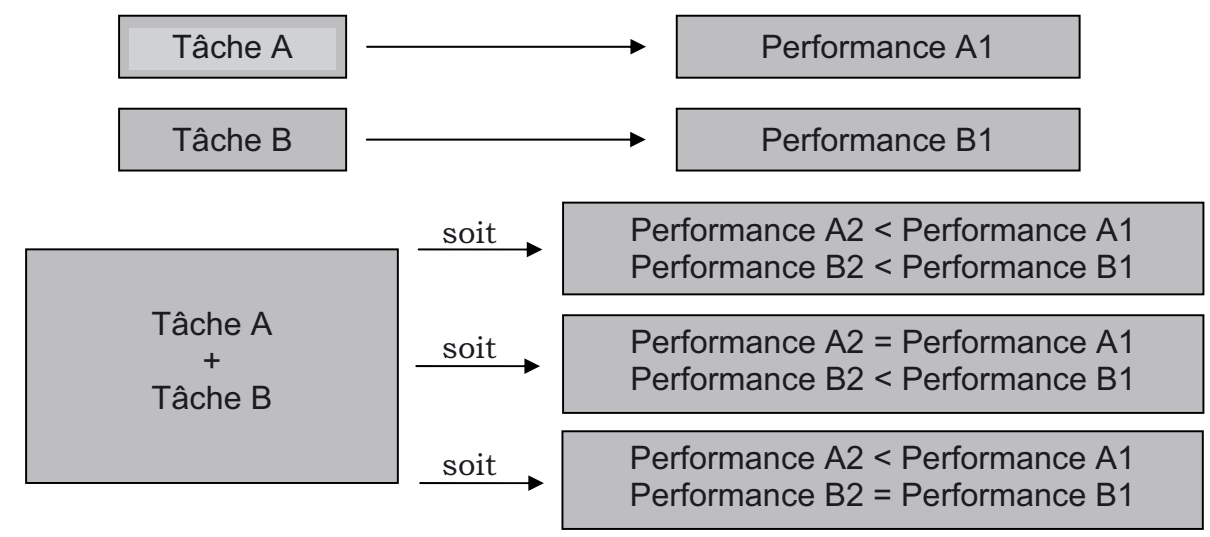

Fig. 2. Illustration schématique d'une situation de double tâche utilisée pour déterminer les stratégies de partage de l'attention.

Mercer, \& Bezodis, 2005) ou les oscillations posturales (Vuillerme \& Nafati, 2007) ont été associées à une augmentation de l'activité neuromusculaire. Cette modulation de l'activité neuromusculaire peut-être interprétée comme une augmentation du recrutement d'unités motrices par le contrôle moteur volontaire, entraînant une augmentation de la force musculaire développée qui à son tour dégrade la précision du contrôle postural lui-même.

Pour tenter de faire une synthèse de ces différents aspects et résultats, l'hypothèse de l'existence d'une relation complexe entre contrôle postural et processus cognitifs peut être avancée. En effet, exécuter une tâche secondaire simple peut améliorer le contrôle postural en déléguant ce dernier à des processus sensori-moteurs de bas niveaux plutôt que de se concentrer sur ces mécanismes de contrôle très automatisés. Dans le même temps, une surcharge des processus cognitifs induite par le traitement de tâches cognitives plus complexes, conduit généralement à une dégradation du contrôle postural.

\section{Effets des informations sensorielles disponibles}

Le contrôle postural est principalement assuré par l'intégration continue des informations sensorielles en provenance des systèmes visuel, proprioceptif, et/ou vestibulaire. Dans ce contexte, quelques travaux se sont intéressés aux effets du contexte sensoriel sur le coût attentionnel du contrôle postural (Shumway-Cook \& Woollacott, 2000; Teasdale \& Simoneau, 2001). Par exemple, Shumway-Cook et Woollacott (2000) ont utilisé une double tâche auditive de temps de réaction de choix dans six contextes sensoriels différents qui modifiaient la disponibilité des informations visuelles et somato-sensorielles pour assurer le contrôle postural. Les résultats ont mis en évidence que chez des sujets jeunes, la double tâche n'affectait pas la stabilité posturale quel que soit le contexte sensoriel manipulé. En revanche, chez des personnes âgées présentant des troubles connus de l'équilibre et des risques de chute, le temps de réaction au- ditif dépendait de ce contexte sensoriel. Quand le contexte sensoriel devenait plus restrictif, les personnes âgées capables de maintenir leur équilibre en situation de simple tâche, présentaient des difficultés en situation de double tâche. Ces données suggèrent que la demande attentionnelle, en fonction de l'âge ( $c f$. les effets de l'âge plus loin) dépend aussi des informations sensorielles disponibles. Dans ce contexte, la capacité d'allouer suffisamment d'attention au contrôle postural peut être un facteur déterminant pour réduire le risque de chute chez des sujets fragiles.

Selon une autre approche, Teasdale et Simoneau (2001) se sont intéressés aux capacités de réintégration (de ré-utilisation) de l'information sensorielle, ainsi qu'au coût attentionnel de ce processus. Pour cela, ces auteurs ont manipulé les informations visuelles et proprioceptives soit en les supprimant, soit en les rendant soudainement disponibles, chez des sujets jeunes et âgés. Dans le même temps, les sujets devaient répondre verbalement et aussi vite que possible à un stimulus auditif. Les résultats ont mis en évidence que la réintégration de l'information proprioceptive, avec ou sans vision disponible, entraînait initialement une perturbation posturale pour les deux groupes. Ces effets étaient plus marqués pour les personnes âgées. Dans le même temps, les auteurs ont observé une augmentation du coût attentionnel du contrôle postural lorsque ces informations proprioceptives étaient réintégrées en absence de vision, quel que soit l'âge des sujets. Ces données mettent en évidence que non seulement le contrôle postural est coûteux attentionnellement, mais également que ce coût dépend des informations sensorielles disponibles et/ou de la nécessité de réintégrer des informations sensorielles initialement absentes.

\section{Effets de la difficulté des tâches cognitive et/ou posturale}

D'une manière générale, la littérature montre que la nature de l'interférence entre contrôle postural et tâche cognitive diffère selon que la tâche cognitive concurrente 
est une tâche verbale (Camicioli, Howieson, Lehman, \& Kaye, 1997; Shumway-Cook et al., 1997), une tâche mathématique (Andersson et al., 2002; Pellecchia, 2003), ou tout autre type de tâche mentale (Dault et al., 2001; Ehrenfried et al., 2003; Melzer et al., 2001). Certains auteurs ont mis en évidence que la tâche posturale avait un effet sur la performance cognitive (Andersson et al., 2002) tandis que d'autres ont montré que la tâche cognitive affectait la stabilité posturale, et cela d'autant plus que la tâche cognitive était complexe à réaliser (Pellecchia, 2003).

Les études dans lesquelles la complexité de la tâche cognitive et celle de la tâche posturale ont été manipulées simultanément sont rares. Par exemple, étudiant le contrôle postural quand la tâche concurrente était une tâche de Stroop (1935), Dault et ses collaborateurs (2001) ont montré une interférence importante lors de l'ajout de cette tâche cognitive à une tâche posturale dont la difficulté était également manipulée via différentes configuration de support. Toutefois, les différentes conditions de Stroop entraînaient toujours le même degré d'interférence (plus la condition de Stroop était complexe et moins les performances posturales étaient bonnes). Plus récemment, des auteurs ont montré que seules les tâches mentales de nature spatiale mais pas les tâches verbales de Stroop, augmentaient le risque de chute de façon significative (Barra, Bray, Sahni, Golding, \& Gresty, 2006). Par ailleurs, ces auteurs n'ont pas observé d'influence de la difficulté de la tâche posturale sur les performances des tâches cognitives manipulées.

Différents auteurs ont montré par ailleurs qu'une tâche posturale a un impact sur les performances cognitives, les tâches posturales les plus difficiles ayant les effets les plus marqués (Andersonn et al., 2002), tandis que d'autres travaux ont montré l'effet inverse (Pellecchia, 2003).

\section{Coût attentionnel dédié au contrôle postural à tous les âges de la vie}

\section{Coût du contrôle postural chez le jeune enfant}

Chez l'enfant, l'hypothèse selon laquelle la capacité attentionnelle augmenterait avec l'âge chronologique a, depuis longtemps déjà, été émise (Wickens, 1974). L'une des premières études à avoir confronté des enfants à une situation de double tâche est celle de Fleury, Bard, \& Nobécourt (1986). Ces auteurs se sont intéressés à la capacité de partager son attention entre une tâche motrice et une tâche verbale cognitive effectuées simultanément. Leurs résultats montrent que les jeunes enfants âgés de 6,7 et 8 ans sont plus perturbés par l'exécution de cette double tâche que les enfants de 11 ans, confirmant ainsi une augmentation des capacités attentionnelles avec l'âge.

Les études s'intéressant au développement de l'attention sélective, c'est-à-dire l'habileté qui consiste à capturer volontairement certains aspects d'un signal et à en ignorer d'autres en fonction de leur degré de pertinence (Enns \& Girgus, 1984), ont envisagé l'existence de deux explications théoriques distinctes. Les ressources attentionnelles mobilisées peuvent être en effet considérées comme : (1) un mécanisme attentionnel unique (Wickens, 1974); ou (2) un ensemble de processus attentionnels indépendants tels qu'une recherche spécifique (overt search), un filtrage des informations (filtering) ou bien une préparation préalable à l'apparition de l'information (priming) (Olivier, Rival, Bard, Fleury, 2003). Dans la théorie du mécanisme attentionnel unique, quelle que soit la nature de la double tâche à exécuter (complexité de la tâche cognitive et/ou de la tâche posturale), le même système attentionnel est sollicité et son efficacité augmente durant l'enfance. Dans le modèle des mécanismes attentionnels multiples, des processus attentionnels différents et indépendants peuvent être sollicités dans différentes situations de double tâche et peuvent avoir une évolution différente au cours de l'enfance.

Concernant l'ontogenèse des performances posturales, de nombreux auteurs observent une amélioration de la stabilité posturale au cours de l'enfance (Kirshenbaum, Riach, \& Starkes, 2001; Sundermier, Woollacott, Roncesvalles, \& Jensen, 2001). Cette amélioration est non-monotonique et caractérisée par un changement drastique de stratégie posturale aux alentours de 7-8 ans (Rival, Ceyte, \& Olivier, 2005). C'est en effet à cet âge que la stratégie adulte correspondant à une coordination entre les mouvements de la tête et du tronc commence à apparaître (Assaiante \& Amblard, 1995). Par ailleurs, entre 4 et 6 ans, le contrôle de la posture est exclusivement basé sur la prise en compte des informations visuelles disponibles alors qu'entre 7 et 10 ans, il est inhérent à une stratégie consistant à également prendre en compte les informations proprioceptives (ShumwayCook \& Woollacott, 1985). Enfin, dans une étude menée auprès d'enfants de $7,8,9,10$ et 11 ans, ainsi que des adultes, il a été récemment montré (Cuisinier, Olivier, Vaugoyeau, Nougier, \& Assaiante, 2011) que dans une situation de perturbation des informations proprioceptives au niveau de la cheville, le contrôle de la posture était très affecté à l'âge de 7 ans alors qu'il ne l'était plus du tout à l'âge adulte. Ainsi, les informations proprioceptives seraient intégrées par le système nerveux central pour contrôler la posture au moins à partir de l'âge de 7 ans, pour être progressivement suppléées par les autres sources d'informations sensorielles entre 7 et 20 ans si nécessaire (i.e., processus de repondération sensorielle). Bien que le développement du contrôle de l'équilibre ait généré un intérêt significatif, les investigations traitant de l'augmentation des ressources attentionnelles dédiées aux fonctions exécutives du contrôle postural avec l'âge restent rares.

Différents travaux dans ce domaine ont, dans un premier temps, tenté de déterminer à quel âge l'enfant est capable d'automatiser le contrôle de sa posture. En effet, l'âge à partir duquel une personne est capable de contrôler sa posture en n'y allouant qu'une toute petite 
quantité de ressources attentionnelles est encore à clarifier. Ainsi, Olivier, Palluel, \& Nougier (2008) ont mené une étude chez des enfants de 4-5 ans, 6-7 ans, 8-9 ans, 10-11 ans ainsi que des adultes, qui consistait à diminuer la quantité de ressources attentionnelles allouées au contrôle orthostatique en orientant l'attention en direction d'un film vidéo (dessin animé). Les résultats obtenus montrent que la stabilité posturale augmente de façon inversement proportionnelle à l'attention exécutive allouée, et ce, dès l'âge de 4-5 ans, suggérant que le processus d'automatisation du maintien d'une posture statique est acquis dès l'âge de 4 ans et devient alors, comme pour l'adulte, plus performant.

D'autres travaux se sont intéressés à l'augmentation ontogénétique des ressources attentionnelles disponibles en fonction de la complexité de la tâche posturale. La littérature montre que, chez l'enfant âgé de 4 à 6 ans, la réalisation simultanée de deux tâches (une cognitive et une posturale) dépasse ses capacités attentionnelles (Laufer, Ashkenazi, \& Josman, 2008; Reilly, Woollacott, van Donkelaar, \& Saavedra, 2008; Reilly, van Donkelaar, \& Saavedra, 2008). Les résultats obtenus par Laufer et al. (2008) montrent en effet que le maintien d'une position orthostatique à l'âge de 5 ans, alors même que ces enfants sont en train de reconnaître et nommer des objets, est fortement dégradé en particulier lorsque la surface d'appui est instable. À cet âge, la réalisation de cette double tâche dépasse les capacités attentionnelles de l'enfant au point de mesurer une dégradation autant dans la tâche posturale que dans la tâche cognitive. Les travaux de Reilly, Woollacott, et al. (2008); Reilly, van Donkelaar, et al., 2008, publiés la même année confirment eux aussi l'existence d'une interférence entre le maintien postural orthostatique et la réalisation simultanée d'une tâche cognitive sollicitant les mécanismes de mémoire de travail entre 4 et 6 ans. Cette interférence est également d'autant plus importante que la posture est complexe. Ainsi, deux études récentes ont été conduites chez des enfants âgés de 7, 8, 9, 10 et 11 ans, ainsi que des adultes. Elles consistaient à maintenir un équilibre orthostatique en position de Romberg décalé sans et avec perturbation posturale (i.e., vibration tendineuse) ainsi que sans et avec tâche cognitive (i.e. tâche de STROOP modifié) associée (Olivier, Cuisinier, Vaugoyeau, Nougier, \& Assaiante, 2007 ; 2010). Les résultats de ces deux études suggèrent qu'à l'âge de 7 ans l'enfant est encore fortement affecté par la réalisation d'une double tâche. Néanmoins, cette dégradation de la performance posturale (passant principalement par une diminution de la vitesse de déplacement du centre des pressions) n'est mesurée que dans la condition où la complexité de la tâche posturale et de la tâche cognitive sont importantes, alors qu'à partir de 8 ans ce n'est plus le cas. En d'autres termes, l'augmentation de l'attention exécutive avec l'âge est non-monotonique et se caractérise par une période charnière se situant autour de 8 ans (Fig. 3).
Ainsi, la complexité de la situation de double tâche proposée aux enfants est déterminante. En effet, les résultats de l'étude menée par Blanchard, Carey, Coffey, Cohen, Michlik, et Pellecchia (2005), portant sur les effets d'interaction entre une tâche cognitive de comptage à rebours ou de lecture et une tâche posturale de maintien orthostatique chez l'enfant de 9 ans et demi, montrent que (1) la présence de cette tâche cognitive altère la stabilité posturale de ces enfants, et que (2) l'augmentation de la complexité de la tâche cognitive engendre, de la part de ces enfants, une adaptation de leur stratégie posturale consistant à diminuer les degrés de liberté possibles en verrouillant certaines articulations. Les résultats obtenus par Schmid, Conforto, Lopez, et D'Alessio (2007) confirment qu'une tâche de compte à rebours exécutée mentalement, i.e. sans mouvement de l'articulation temporo-mandibulaire qui pourrait avoir une incidence sur la posture, perturbe de façon importante les stratégies posturales d'un enfant âgé de 9 ans. Ces auteurs suggèrent que l'adjonction d'une tâche cognitive complexe à une tâche posturale dépasse les capacités attentionnelles de ces enfants.

En conclusion, les connaissances actuelles nous amènent à penser que les enfants sont régulièrement confrontés à des situations de la vie de tous les jours qui dépassent leur capacité attentionnelle et les mettent en position d'échec. Par exemple, à l'école, réciter un poème ou une récitation debout devant le tableau alors qu'ils seraient mieux assis. Ou encore lorsqu'ils sont en cours de gymnastique en train de marcher sur une poutre et que l'enseignant leur parle.

\section{Coût du contrôle postural chez l'adolescent}

La puberté, étape du développement humain marquant la transition de l'enfance à l'adolescence (fertilité), débute aux alentours de 11 ans (âge biologique) chez les filles et 13 ans chez les garçons. Elle se caractérise par un développement rapide en termes de taille, forme et composition corporelle. Cependant, ces changements n'impliquent pas une dégradation du contrôle postural. En effet, des études ont montré une amélioration de la stabilité posturale entre 5 et 18 ans avec des changements minimes entre 8 et 18 ans (Ferber-Viart, Ionescu, Morlet, Froehlich, \& Dubreuil, 2007; Mallau, Vaugoyeau, \& Assaiante, 2010; Wolff, Rose, Jones, Bloch, Oehlert, \& Gamble, 1998). Ferber-Viart et al. (2007), en se basant sur l'Equitest, indiquent que le comportement adulte n'est pas atteint à l'âge de 12 ans. Nolan, Grigorenko, et Thorstensson (2005) suggèrent que l'évolution du contrôle postural avec l'âge est liée au développement des systèmes visuel, vestibulaire et somatosensoriel. Par exemple, nous savons que les adolescents de 14-15 ans négligent temporairement les informations proprioceptives au profit des informations visuelles lors du contrôle de l'orientation et de la stabilisation corporelle (Viel, Vaugoyeau, \& Assaiante, 2009), les 


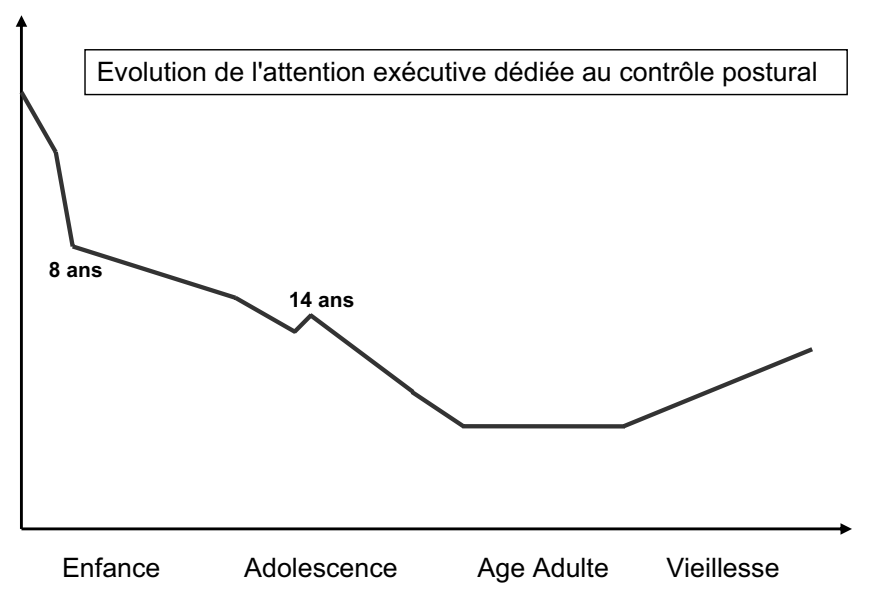

Fig. 3. Représentation schématique de l'évolution de la demande en attention exécutive dédiée au contrôle postural aux différents âges de la vie.

performances posturales des garçons étant particulièrement affectées.

Les ressources attentionnelles mobilisées lors du contrôle postural chez l'adolescent de 12 à 17 ans ont récemment été évaluées par Palluel et al. (2010) dans un paradigme de double tâche. La tâche posturale primaire consistait à maintenir un équilibre en position semitandem pendant $30 \mathrm{~s}$ sur une surface dure ou sur de la mousse. Différentes tâches cognitives secondaires (Stroop et comptage de 3 en 3 à rebours) ont été réalisées simultanément à la tâche posturale. Ces auteurs n'ont pas observé de différence entre les filles et les garçons, ni d'effet de la surface de support sur les performances posturales et cognitives. Néanmoins, une période charnière a été observée entre 14 et 15 ans lors d'une situation de double tâche. Les performances posturales étaient moins bonnes à cet âge, quelle que soit la tâche cognitive. Ces résultats confirment ceux de Viel et al. (2009) dans une situation de simple tâche posturale. La dégradation était particulièrement marquée lors du compte à rebours de 3 en 3. Cette tâche requiert plus de ressources attentionnelles que les autres tâches (Blanchard et al., 2005). Cette différence observée à 14-15 ans peut certainement s'expliquer par la difficulté des adolescents à mobiliser efficacement les ressources attentionnelles nécessaires à la réalisation de deux tâches concomitantes en raison du passage d'une étape développementale à une autre.

Pour conclure, l'unique étude réalisée sur le coût attentionnel de la posture chez l'adolescent par Palluel, Nougier, et Olivier (2010) suggère qu'il existerait une deuxième période charnière aux alentours de $14-15$ ans puisque le contrôle postural se dégrade à cet âge en situation de double tâche et n'a donc pas atteint sa pleine maturité (Fig. 3).

\section{Coût du contrôle postural chez la personne âgée}

Si l'on s'intéresse maintenant à la population des personnes âgées, cette dernière se caractérise par une dégénérescence des récepteurs proprioceptifs musculaires (Swash \& Fox, 1972; Kararizou, Manta, Kalfakis, \& Vassilopoulos, 2005; Liu, Eriksson, Thornell, \& PedrosaDomellof, 2005; Rosant et al., 2007), articulaires (Salo \& Tatton, 1993; Morisawa, 1998; Aydoğ, Korkusuz, Doral, Tetik, \& Demirel, 2006) et cutanés (Cauna \& Mannan, 1958; Bolton, Winkelmann, \& Dyck, 1966), ainsi que des récepteurs vestibulaires (Richter, 1980). Ces modifications sont physiologiquement liées au vieillissement. Elles conduisent à une diminution de la qualité et de l'intensité des signaux sensoriels utiles au maintien d'une posture stable (Levin \& Benton, 1973; Miwa, Miwa, \& Kanda, 1995). L'altération de ces signaux à un niveau périphérique pourrait se traduire par une augmentation du coût attentionnel requis pour leur traitement à un niveau central. De plus, ce phénomène pourrait être amplifié par la dégradation des processus cognitifs liée au vieillissement (Grady \& Craik, 2000) qui ont par ailleurs montré avoir une interdépendance renforcée avec les processus sensoriels et sensori-moteurs chez les personnes âgées (Li \& Dinse, 2002; Li \& Lindenberger, 2002). Plusieurs études se sont intéressées à ce phénomène par le biais de différents paradigmes de double tâche.

Alors que chez des sujets adultes sains peu de ressources attentionnelles sont nécessaires pour contrôler la posture, ce coût attentionnel augmente chez les personnes âgées (Woollacott \& Shumway-Cook, 2002). En effet de nombreuses études ont montré, dans des conditions de double tâche, une dégradation de la performance dans la tâche secondaire chez des personnes âgées non observée chez de jeunes adultes (Brown, Sleik, \& Winder, 2002; Mahboobina, Loughlin, \& Redfern, 2007; Maylor \& Wing, 1996; Tucker, Kavanagh, Barrett, \& Morrison, 2008; Gage, Sleik, Polych, McKenzie, \& Brown, 2003; Teasdale \& Simoneau, 2001; Marsh \& Geel, 2000). Une dégradation a également été mesurée pour une population de personnes âgées entre une posture simple (par exemple assise) et une posture plus complexe (Teasdale \& Simoneau, 2001; Lajoie \& Gallagher, 2004; Lajoie et al., 1996), ou encore entre la phase de simple et de double appui au cours de la marche (Lajoie, Teasdale, Bard, \& Fleury, 1993). De plus, Teasdale, Bard, LaRue, \& Fleury (1993) ont également montré qu'une réduction des informations sensorielles disponibles augmente la demande attentionnelle posturale chez les sujets âgés par rapport aux sujets jeunes. Dans la lignée de ces résultats, BernardDemanze et al. (2009) ont montré que le coût attentionnel de la double tâche était dépendant de la complexité de la tâche cognitive et de la difficulté de la tâche posturale.

Certains auteurs ont établi une corrélation positive entre l'altération de la performance dans la tâche secondaire et l'augmentation du risque de chute chez les personnes âgées (Gage et al., 2003; Tucker, Kavanagh, Morrison, \& Barrett, 2009). De ce fait, pour pouvoir 
réaliser plusieurs tâches à la fois sans prendre de risque, certaines personnes âgées pourraient être limitées à un nombre de stimulations environnementales restreintes, dans lesquelles les conditions sensorielles sont optimales. Par exemple, ne pas devoir penser à éviter de se prendre les pieds dans le tapis pour aller répondre au téléphone.

La demande attentionnelle relative à la tâche réalisée semble également influencer le choix de la réponse motrice mise en place pour maintenir une posture stable. Brown, Shumway-Cook, et Woollacott (1999) suggèrent que les stratégies motrices utilisées (pieds-en-place vs. déplacement d'un pied) par les sujets sont choisies en fonction de la demande attentionnelle. Pour les sujets jeunes et âgés, le déplacement d'un pied survient lorsque la tâche secondaire est ajoutée et cet effet est accentué chez la personne âgée. Pourtant Brown et al. (1999) ont également montré que le déplacement d'un pied est une stratégie plus coûteuse d'un point de vue attentionnel pour les personnes âgées, et son utilisation dans une situation plus complexe pourrait en fait accentuer cette complexité de la tâche et dépasser les ressources attentionnels nécessaires à une posture stable. La diminution de l'amplitude de la réponse des muscles posturaux chez la personne âgée, mise en évidence par Rankin et al. (2000), pourrait obliger la mise en place d'une stratégie de déplacement-d'un-pied précoce dans des situations de rééquilibration malgré le surcoût occasionné en termes d'attention. Bernard-Demanze, Dumitrescu, Jimeno, Borel, et Lacour (2009) ont montré que les stratégies mises en œuvre pour récupérer l'équilibre dans une tâche posturale dynamique est différente pour la personne âgée et la personne jeune. Cette différence pourrait prendre sa source dans une différence de priorité mise en évidence entre les personnes jeunes et âgées. De récentes études (Bernard-Demanze et al., 2009; Berger \& Bernard-Demanze, 2011; Riley, Baker, Schmit, \& Weaver, 2005) ont montré que les sujets jeunes améliorent leur performance dans un contexte de double tâche alors que les personnes âgées la diminue. Ces auteurs expliquent ces résultats par un principe de « cognition en premier » chez les sujets jeunes, qui diffère du principe de « posture en premier $»$ mis en évidence chez les sujets âgés (Shumway-Cook et al., 1997).

Cette augmentation du coût attentionnel observé chez les personnes âgées par rapport aux personnes jeunes dans un but de maintenir une performance posturale stable renvoie à des études récentes (Heuninckx, Wenderoth, Debaere, Peeters, \& Swinnen, 2005; Heuninckx, Wenderoth, \& Swinnen, 2008, 2010; Hutchinson et al., 2002; Mattay et al., 2002; Naccarato, Calautti, Jones, Day, Carpenter, \& Baron, 2006) qui montrent que les personnes âgées recrutent des ressources neurales additionnelles afin d'atteindre un niveau de performance motrice comparable à celui obtenu par des sujets jeunes.

En conclusion, cette revue de littérature traitant de l'attention et du contrôle postural chez des sujets âgés sains démontre (1) que le contrôle postural nécessite davantage d'attention chez la personne âgée (Fig. 3) ; que (2) la réalisation d'une tâche secondaire qui nécessite de l'attention produit un effet délétère plus important sur le contrôle postural de la personne âgée que sur celui du sujet jeune; (3) que les effets de la tâche secondaire sur le contrôle postural dépendent de plusieurs facteurs tels que la complexité de la tâche secondaire et la difficulté de la tâche posturale; et que (4) cette augmentation du coût attentionnel lié au contrôle postural observé chez la personne âgé par rapport au sujet jeune pourrait rendre compte d'une augmentation des ressources neurales mises en jeu pour maintenir une posture stable.

\section{Coût attentionnel du contrôle postural et handicap ou expertise}

De la même manière que pour le vieillissement physiologique, de nombreuses pathologies affectent le comportement posturo-cognitifs des patients. Que ces maladies affectent directement ou indirectement les corrélats neuronaux impliqués dans le contrôle de la posture ou des activités motrices, les résultats de nombreuses études s'accordent à dire que les ressources attentionnelles allouées à ce contrôle sont davantage sollicitées chez les personnes fragiles que chez des individus sains. Ces différentes investigations se basent sur des protocoles de double tâche. Parallèlement à la dégradation de la performance à la tâche cognitive, des déficits de stabilité posturale et/ou d'habilité motrice sont souvent observés et assimilés à une augmentation du risque de chute ainsi que de la cascade de problèmes associés. Par exemple, Reilly, Woollacott, et al. (2008) ont étudié la stabilité posturale au cours d'un paradigme de double tâche chez des enfants atteints d'infirmité motrice cérébrale. Les auteurs notent que les performances aux tâches posturales et cognitive (mémorisation de stimuli visuels) du groupe de huit enfants infirmes moteurs cérébraux âgés de 10 à 14 ans sont fortement dégradées par rapport à celles des enfants sains du même âge et identiques à celles d'enfants sains âgés de 4 à 6 ans.

Les patients atteints de maladies neuro-dégénératives de type Parkinson ou Alzheimer sont fréquemment victimes de chute, favorisant l'aggravation de la dépendance. Ces troubles de l'équilibre peuvent être imputés à une défaillance dans le contrôle de la posture et/ou de la locomotion. Certaines études ont étudié les caractéristiques spatio-temporelles de la marche au cours de situations de double tâche, mêlant à la contrainte motrice une sollicitation des ressources attentionnelles. Ainsi, certains auteurs ont montré que les individus atteints par la maladie d'Alzheimer réduisent significativement leur vitesse de marche (tout en augmentant la variabilité de celles-ci au cours des essais) lorsqu'une contrainte cognitive (répétition de chiffres mémorisés) est adjointe à la tâche locomotrice (Sheridan, Solomont, Kowall, \& Hausdorff, 2003). Les patients déments souffrent d'une difficulté à associer les 
fonctions exécutives de la marche et les fonctions neuropsychologiques essentielles au traitement des informations extra-motrices. Selon les auteurs, ce conflit serait à l'origine de l'augmentation du risque de chute pour cette population. Avec un protocole identique, Yogev, Giladi, Peretz, Springer, Simon, et Hausdorff (2005) ont conduit une étude chez les individus souffrant de la maladie de Parkinson. Alors que tous les sujets évalués présentaient une réduction de la vitesse de marche entre les conditions de simple et de double tâche, cette différence était significativement plus importante chez les patients parkinsoniens. Alors que pour les sujets sains, la marche peut être considérée comme une activité relativement automatisée, ne sollicitant pas de manière importante les ressources attentionnelles, pour les patients parkinsoniens, la disparition progressive des neurones qui produisent la dopamine, ce neurotransmetteur qui commande la motricité automatique, se traduit par une demande cognitive plus importante qui participe à l'augmentation du risque de chute chez ces patients.

Outre les aspects dégénératifs, les accidents neurologiques de type mécaniques (blessés médullaires) ou ischémiques (accident vasculaire cérébraux (AVC)) ont également fait l'objet d'études comparables à celles précédemment évoquées. Ainsi, Lajoie et al. (1999) ont étudié le temps de réaction à des stimuli sonores lors de quatre tâches posturales ou motrices (assis, debout en condition bipodale, marche à vitesse libre et marche à vitesse imposée) chez un groupe de patients avec une lésion partielle de la moelle épinière et un groupe de sujets sains. Alors que pour les tâches statiques (assis et maintien de la posture érigée sur deux pieds) les temps de réaction sont plus rapides pour le groupe de patients blessés médullaires, le sens de la performance s'inverse lors de la locomotion. La marche des patients blessés médullaires requiert davantage de ressources attentionnelles que les sujets sains et ce, davantage lorsque le stimulus est présenté au cours de la phase de simple appui pendant le cycle de marche.

De la même manière, Brown et al. (2002) ont comparé les temps de réaction verbaux à la présentation de stimuli visuels lors de trois tâches posturales (assis, debout deux pieds écartés et debout deux pieds serrés) chez un groupe de patients ayant eu une attaque ischémique cérébrale récente (AVC) (moins d'un an) et un groupe de sujets sains du même âge (64 ans). Alors que le temps de réaction reste inchangé pour le groupe de sujets sains quelle que soit la tâche posturale, ce temps de réaction est fortement accru quand la complexité de la tâche posturale augmente chez le groupe de patients AVC. D'autres pathologies n'affectant pas directement le système nerveux requièrent des coûts attentionnels importants au cours de certaines activités posturales. Par exemple, l'obésité, maladie épidémique dont la prévalence est en constante augmentation depuis plus de deux décennies, affecte non seulement un grand nombre de caractéristiques physiologiques, psychologiques et sociales mais engendre également des désordres du contrôle postural et des habiletés motrices (Maffiuletti et al., 2007, Berrigan, Simoneau, Tremblay, Hue, \& Teasdale, 2006, Bray, 2004). D'un point de vue neurophysiologique, il a été montré que le schéma corporel, essentiel à la conception des modèles internes pour l'action, est erroné chez les obèses (Mignardot et al., 2010). En effet, la représentation qu'un sujet se fait de son corps se construit à partir des informations issues des organes somato-sensoriels. Or, ces auteurs suggèrent que la qualité et la quantité des influx nerveux en provenance de ces organes, peuvent être altérées par les contraintes morphologiques et par le style de vie sédentaire relatif à cette pathologie. Les résultats de Mignardot, Olivier, Promayon, et Nougier (2010) ont montré que les obèses souffraient d'une grande difficulté à maintenir une posture érigée sur un pied et que les fortes oscillations du centre des pressions (CP) allaient régulièrement jouer avec les limites géographiques de la surface d'appui. Face à un groupe de sujets non obèses du même âge (45 ans), les oscillations posturales sont deux fois plus importantes et ce, que les sujets soient en situation de simple tâche (posture seulement) ou de double tâche (posture + temps de réaction à des stimuli sonores). Alors que le temps de réaction est inchangé chez le groupe de non obèses entre une condition posturale simple (assis) ou complexe (unipodale), il augmente très fortement chez le groupe obèse. Dans cette étude, aucune instruction n'était donnée aux sujets quand à la priorité à donner à la tâche posturale ou réactionnelle. Chez les sujets obèses, l'absence de différence posturale entre les conditions de simple et de double tâche et l'augmentation du temps de réaction concomitante en situation unipodale met en évidence la priorité donnée par les obèses au contrôle postural. Au regard des très fortes oscillations du $\mathrm{CP}$, si ces patients réduisaient le coût attentionnel dédié au contrôle postural, il est probable qu'ils chuteraient. C'est pourquoi les obèses préfèrent réduire leur performance à la tâche qu'ils considèrent comme secondaire et qui n'affecte pas leur intégrité physique. Cependant, dans la vie de tous les jours, nombreuses sont les situations où il est nécessaire de gérer des contraintes cognitives en parallèle de contraintes posturales ou locomotrices. Les résultats de cette étude montrent clairement que les sujets obèses ne sont pas en mesure d'être performants en situation de multi-tâches lorsque les sujets sains peuvent l'être.

Pour les individus souffrant d'une pathologie atteignant directement (infirmité motrice cérébrale, maladie neuro-dégénérative, section de la moelle épinière, accident vasculaire cérébral) ou indirectement (exemple de l'obésité) les corrélats neuronaux contrôlant le mouvement, le maintien debout ou les activités locomotrices représentent un réel challenge cognitif qui œuvre en défaveur de l'aisance à préserver son intégrité physique et/ou à répondre à des sollicitations extérieures (e.g., répondre à une personne qui vous questionne).

Si le handicap cognitif et/ou moteur affecte le coût du contrôle postural, les effets de l'expertise sur le coût attentionnel du contrôle de la posture peuvent également être considérés. En effet, certaines habiletés sportives telles 


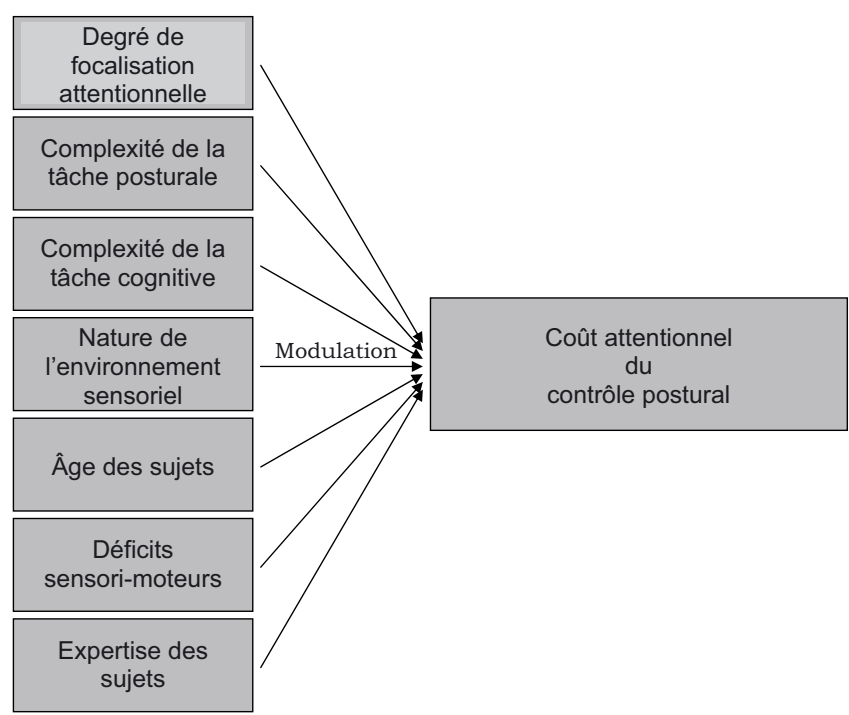

Fig. 4. Représentation schématique des principaux facteurs susceptibles de moduler le coût attentionnel du contrôle postural.

que la gymnastique ou les épreuves de tir (tir à l'arc, tir au pigeon, etc.) imposent un contrôle postural très fin. Les études restent peu nombreuses dans ce domaine mais conduiraient sans doute à des résultats similaires. Par exemple, Vuillerme \& Nougier (2004) se sont intéressés à la capacité de gymnastes experts et de sportifs non experts à contrôler leur posture dans des situations de complexité posturale croissante tout en exécutant simultanément une tâche de temps de réaction. Si les résultats mettent en évidence une augmentation du coût attentionnel (du temps de réaction) avec la complexité de la tâche posturale, cette augmentation s'avère plus limitée pour les gymnastes experts, suggérant un contrôle postural plus efficace et donc moins coûteux attentionnellement.

\section{Conclusion}

Comme on aura pu le constater au travers de cette rapide revue de la littérature, les relations entre contrôle postural et coût attentionnel sont complexes et parfois difficiles à synthétiser. Les facteurs en jeu sont multiples, tels que la nature de la tâche ajoutée, sa complexité associée à la complexité de la tâche posturale, le caractère prioritaire ou non de la tâche cognitive ou posturale, la nature des informations sensorielles disponibles, l'âge des sujets ou plus généralement leurs capacités sensorimotrices (Fig. 4).

Par conséquent, selon comment ces facteurs sont manipulés ou interagissent, les effets observés semblent parfois contradictoires et d'interprétation difficile. Toutefois, certaines règles ou principes peuvent être dégagés. D'une manière générale, on peut conclure que le coût attentionnel du contrôle postural est d'autant plus élevé que (1) la focalisation sur ce contrôle postural est élevée, (2) la complexité de la tâche posturale et/ou cognitive est élevée, (3) que l'environnement sensoriel est pauvre et/ou que la réintégration des informations sensorielles disponibles est complexe, (4) les sujets sont jeunes, en cours de développement, ou subissent au contraire les effets du vieillissement, (5) les sujets présentent des troubles posturaux liés à un déficit sensori-moteur, de quelque nature que ce soit. Au-delà de ces résultats expérimentaux, il reste toutefois que les supports neurologiques et la nature exacte des processus mentaux sous-jacents restent encore mal connus.

\section{Bibliographie}

Abernethy, B. (1988). Dual-task methodology and motor skills research : some methodological constraints. Journal of Human Movement Studies, 14, 101-132.

Andersson, G., Hagman, J., Talianzadeh, R., Svedberg, A., \& Larsen, H.C. (2002). Effect of cognitive load on postural control. Brain Research Bulletin, 58, 135-139.

Andersson, G., Yardley, L., \& Luxon, L. (1998). A dual-task study of interference between mental activity and control of balance. American Journal of Otolaryngology, 19, 632637.

Assaiante, C., \& Amblard, B. (1995). An ontogenetic model for the sensorimotor organization of balance control in humans. Human Movement Science, 14, 13-43.

Aydoğ, S.T., Korkusuz, P., Doral, M.N., Tetik, O., \& Demirel, H.A. (2006). Decrease in the numbers of mechanoreceptors in rabbit ACL: the effects of ageing. Knee Surgery, Sports Traumatology, Arthroscopy, 14, 325-329.

Barra, J., Bray, A., Sahni, V., Golding, J.F., \& Gresty, M.A. (2006). Increasing cognitive load with increasing balance challenge: recipe for catastrophe. Experimental Brain Research, 174, 734-745.

Belenkii, V.E., Gurfinkel, V.S., \& Paltsev, E.I. (1967). On elements of control of voluntary movements. Biofizica, 12, 135-141.

Berger, L., \& Bernard-Demanze, L. (2011). Age-related effects of a memorizing spatial task in the adults and elderly postural control. Gait \&5 Posture, 33, 300-302.

Berrigan F., Simoneau M., Tremblay A., Hue O., \& Teasdale N. (2006). Influence of obesity on accurate and rapid arm movement performed from a standing posture. International Journal of Obesity 30, 1750-1757.

Bernard-Demanze, L., Dumitrescu, M., Jimeno, P., Borel, L., \& Lacour, M. (2009). Age-related changes in posture control are differentially affected by postural and cognitive task complexity. Current Aging Science, 2, 139-149.

Blanchard, Y., Carey, S., Coffey, J., Cohen, A., Michlik, S., \& Pellecchia, G.L. (2005). The influence of concurrent cognitive tasks on postural sway in children. Pediatric Physical Therapy, 17, 189-193. 
Bolton, C.F., Winkelmann, R.K., \& Dyck, P.J. (1966). A quantitative study of Meissner's corpuscles in man. Neurology, 16, 1-9.

Bourke, P.A., Duncan, J., \& Nimmo-Smith, I. (1996). A general factor involved in dual-task performance decrement. Quaterly Journal of Experimental Psychology: Section AHuman Experimental Psychology, 49, 525-545.

Bray, G.A. (2004) Medical Consequences of Obesity. The Journal of Clinical Endocrinology \& Metabolism, 89, 25832589.

Brown, L.A., Shumway-Cook, A., \& Woollacott, M.H. (1999). Attentional demands and postural recovery: the effects of aging. Journal of Gerontology, 54, 165-171.

Brown, L.A., Sleik, R.J., \& Winder, T.R. (2002). Attentional demands for static postural control after stroke. Archives of Physical Medicine and Rehabilitation, 83, 1732-1735.

Camicioli, R., Howieson, D., Lehman, S., \& Kaye, J. (1997). Talking while walking: the effect of a dual task in aging and Alzheimer's disease. Neurology, 48, 955-958.

Cauna, N., \& Mannan, G. (1958). The structure of human digital pacinian corpuscles (corpuscula lamellosa) and its functional significance. Journal of Anatomy, 92, 1-20.

Cuisinier, R., Olivier, I., Vaugoyeau, M., Nougier, V., \& Assaiante, C. (2011). Development of proprioceptive inputs using for postural control in children from 7 to 11 and adults. Plos One, 6, e19697.

Dault, M.C., Geurts, A.C.H., Mulder, T.W., \& Duysens, J. (2001). Postural control and cognitive task performance in healthy participants while balancing on different supportsurface configurations. Gait \& Posture, 14, 248-255.

Ehrenfried, T., Guerraz, M., Thilo, K.V., Yardley, L., \& Gresty, M.A. (2003). Posture and mental task performance when viewing a moving visual field. Cognitive Brain Research, 17, 140-153.

Enns, J.T., \& Girgus, J.S. (1984). Developmental changes in selective and integrative visual attention. Journal of Experimental Child Psychology, 40, 319-337

Ferber-Viart, C., Ionescu, E., Morlet, T., Froehlich, P., \& Dubreuil, C. (2007). Balance in healthy individuals assessed with Equitest: maturation and normative data for children and young adults. International Journal of Pediatrics and Otorhinolaryngology, 71, 1041-1046.

Fleury, M., Bard, C., \& Nobécourt, P. (1986). Ontogenetic study of time sharing in children aged 6-11. Journal of Human Movement Studies, 12, 259-269.

Gage, W.H., Sleik, R.J., Polych, M.A., McKenzie, N.C., \& Brown, L.A. (2003). The allocation of attention during locomotion is altered by anxiety. Experimental Brain Research, 150, 385-394.

Grady, C.L., \& Craik, F.I. (2000). Changes in memory processing with age. Current Opinion in Neurobiology, 10, 224-231.

Heuninckx, S., Wenderoth, N., Debaere, F., Peeters, R., \& Swinnen, S.P. (2005). Neural basis of aging: the penetration of cognition into action control. Journal of Neuroscience, 25, 6787-6796.
Heuninckx, S., Wenderoth, N., \& Swinnen, S.P. (2008). Systems neuroplasticity in the aging brain: recruiting additional neural resources for successful motor performance in elderly persons. Journal of Neuroscience, 28, 91-99.

Heuninckx, S., Wenderoth, N., \& Swinnen, S.P. (2010). Agerelated reduction in the differential pathways involved in internal and external movement generation. Neurobiology of Aging, 31, 301-314.

Hutchinson, S., Kobayashi, M., Horkan, C.M., Pascual-Leone, A., Alexander, M.P., \& Schlaug, G. (2002). Age-related differences in movement representation. Neuroimage, 17, 1720-1728.

Kararizou, E., Manta, P., Kalfakis, N., \& Vassilopoulos, D. (2005). Morphometric study of the human muscle spindle. Analytical and Quantitative Cytology 83 Histology, 27, 1-4.

Kerr, B., Condon, S.M., \& McDonald, L.A. (1985). Cognitive spatial processing and the regulation of posture. Journal of Experimental Psychology Human Perception and Performance, 11, 617-622.

Kirshenbaum, N., Riach, C.L., \& Starkes, J.L. (2001). Nonlinear development of postural control and strategy use in young children: a longitudinal study. Experimental Brain Research, 140, 420-431.

Lajoie, Y., Barbeau, H., \& Hamelin, M. (1999). Attentional requirements of walking in spinal cord injured patients compared to normal subjects. Spinal Cord, 37, 245-250.

Lajoie, Y., \& Gallagher, S.P. (2004). Predicting falls within the elderly community: comparison of postural sway, reaction time, the Berg balance scale and the Activitiesspecific Balance Confidence (ABC) scale for comparing fallers and non-fallers. Archives of Gerontology and Geriatrics, 38, 11-26.

Lajoie, Y., Teasdale, N., Bard, C., \& Fleury, M. (1993). Attentional demands for static and dynamic equilibrium. Experimental Brain Research, 97, 139-144.

Lajoie, Y., Teasdale, N., Bard, C., \& Fleury, M. (1996). Upright standing and gait: are there changes in attentional requirements related to normal aging? Experimental Aging Research, 22, 185-198.

Laufer, Y., Ashkenazi, T., \& Josman, N. (2008). The effects of a concurrent cognitive task on the postural control of young children with and without developmental coordination disorder. Gait \&S Posture, 27, 347-351.

Levin, H.S., \& Benton, A.L. (1973). Age effects in proprioceptive feedback performance. Gerontologia Clinica, 15, 161-169.

Li, K.Z., \& Lindenberger, U. (2002). Relations between aging sensory/sensorimotor and cognitive functions. Neuroscience \& Biobehavioral Reviews, 26, 777-783.

Li, S.C., \& Dinse, H.R. (2002). Aging of the brain, sensorimotor, and cognitive processes. Neuroscience $\&$ Biobehavioral Reviews, 26, 729-732.

Liu, J.X., Eriksson, P.O., Thornell, L.E., \& Pedrosa-Domellof, F. (2005). Fiber content and myosin heavy chain composition of muscle spindles in aged human biceps brachii. 
Journal of Histochemistry and Cytochemistry, 53, 445454 .

Maffiuletti, N.A., Jubeau. M.M., Munzinger, U., Bizzini, M., Agosti, F., et al. (2007). Differences in quadriceps muscle strength and fatigue between lean and obese subjects. European Journal of Applied Physiology, 101, 51-59.

Mahboobina, A., Loughlin, P.J., \& Redfern, M.S. (2007). A model-based approach to attention and sensory integration in postural control of older adults. Neuroscience Letters, 429, 147-151.

Maki, B.E., \& McIlroy, W.E. (1996). Influence of arousal and attention on the control of postural sway. Journal of Vestibular Research, 6, 53-59.

Mallau, S., Vaugoyeau, M., \& Assaiante, C. (2010). Postural strategies and sensory integration: no turning point between childhood and adolescence. PLoS One, 5, e13078.

Marsh, A.P., \& Geel, S.E. (2000). The effect of age on the attentional demands of postural control. Gait \& Posture, 12, 105-113.

Massion, J. (1992). Movement, posture and equilibrium Interaction and coordination. Progress in Neurobiology, $38,35-56$.

Mattay, V.S., Fera, F., Tessitore, A., Hariri, A.R., Das, S., Callicott, J.H., \& Weinberger, D.R. (2002). Neurophysiological correlates of age-related changes in human motor function. Neurology, 58, 630-635.

Maylor, E.A., \& Wing, A.M. (1996). Age differences in postural stability are increased by additional cognitive demands. Journal of Gerontology, 51B, P143-154.

Melzer, I., Benjuya, N., \& Kaplanski, J. (2001). Age-related changes of postural control: Effect of cognitive tasks. Gerontology, 47, 189-194.

Mignardot, J.B., Olivier, I., Promayon, E., \& Nougier, V. (2010). Obesity impact on the attentional cost for controlling posture. PLoS One, 5, e14387.

Miwa, T., Miwa, Y., \& Kanda, K. (1995). Dynamic and static sensitivities of muscle spindle primary endings in aged rats to ramp stretch. Neuroscience Letters, 201, 179-182.

Morisawa, Y. (1998). Morphological study of mechanoreceptors on the coracoacromial ligament. Journal of Orthopaedic Science, 3, 102-110.

Naccarato, M., Calautti, C., Jones, P.S., Day, D.J., Carpenter, T.A., \& Baron, J.C. (2006). Does healthy aging affect the hemispheric activation balance during paced indexto-thumb opposition task? An fMRI study. Neuroimage, 32, 1250-1256.

Nolan, L., Grigorenko, A., \& Thorstensson, A. (2005). Balance control : sex and age differences in 9- to 16-year-olds. Developmental Medicine and Child Neurology, 47, 449454.

Olivier, I., Cuisinier, R., Vaugoyeau, M., Nougier, V., \& Assaiante, C. (2007). Dual-task study of cognitive and postural interference in 7-year-old and adults. Neuroreport, 18, 817-821.
Olivier, I., Cuisinier, R., Vaugoyeau, M., Nougier, V., \& Assaiante, C. (2010). Age-related differences in cognitive and postural dual-task performance. Gait \& Posture, 32, 494-499.

Olivier, I., Palluel, E., \& Nougier, V. (2008). Effects of attentional focus on postural sway in children. Experimental Brain Research, 185, 341-345.

Olivier, I., Rival, C, Bard, C., \& Fleury, M. (2003). Agedrelated differences in the Attentional cost of pointing movements. Neuroscience Letters, 338, 169-173.

Palluel, E., Nougier, V., \& Olivier, I. (2010). Postural control and attentional demand during adolescence. Brain Research, 1358, 151-159.

Pellecchia, G.L. (2003). Postural sway increases with attentional demands of concurrent cognitive task. Gait $\&$ Posture, $18,29-34$.

Rankin, J.K., Woollacott, M., Shumway-Cook, A., \& Brown, L. (2000). A neuromuscular analysis of the influence of a cognitive task on postural stability in young and older adults. Journal of Gerontology Series A: Biological Sciences and Medical Sciences, 55, M112-119.

Reilly, D.S., van Donkelaar, P., \& Saavedra, S. (2008). The interaction between the development of postural control and the executive function of attention. Journal of Motor Behavior, 40, 90-102.

Reilly, D.S., Woollacott, M.H., van Donkelaar, P., \& Saavedra, S. (2008). The interaction between executive attention and postural control in dual-task conditions: Children with cerebral palsy. Archives of Physical Medicine and Rehabilitation, 89, 834-842.

Richter E. (1980). Quantitative study of human Scarpa's ganglion and vestibular sensory epithelia. Acta Otolaryngologica, 90, 199-208.

Riley, A., Baker, A.A., Schmit, J., \& Weaver, E. (2005). Effects of visual and auditory short-term memory tasks on the spatiotemporal dynamics and variability of postural sway. Journal of Motor Behavior, 37, 311-324.

Rival, C., Ceyte, H., \& Olivier, I. (2005). Developmental changes of static standing balance in children. Neuroscience Letters, 376, 133-136.

Rosant, C., Nagel, M.D., \& Pérot, C. (2007). Aging affects passive stiffness and spindle function of the rat soleus muscle. Experimental Gerontology, 42, 301-308.

Salo, P.T., \& Tatton, W.G. (1993). Age-related loss of knee joint afferents in mice. Journal of Neuroscience Research, $35,664-677$.

Schmid, M., Conforto, S., Lopez, L., \& D'Alessio, T. (2007). Cognitive load affects postural control in children. Experimental Brain Research, 179, 375-85.

Sheridan, P.L., Solomont, J., Kowall, N., \& Hausdorff, J.M. (2003). Influence of executive function on locomotor function: divided attention increases gait variability in Alzheimer's disease. Journal of American Geriatric Society, 51, 1633-1637. 
Shumway-Cook, A., Woollacott, M.A. (1985). The growth of stability : postural control from a development perspective. Journal of Motor Behavior, 17, 131-147.

Shumway-Cook, A., \& Woollacott, M.H. (2000). Attentional demands and postural control: the effect of sensory context. Journal of Gerontology Series A: Biological Sciences and Medical Sciences, 55, M10-M16.

Shumway-Cook, A., Woollacott, M., Kerns, K.A., \& Baldwin, M. (1997). The effects of two types of cognitive tasks on postural stability in older adults with and without a history of falls. Journal of Gerontology Series A: Biological Sciences and Medical Sciences, 52, M232-M240.

Stelmach, G.E., Zelaznik, H.N., \& Lowe, D. (1990). The influence of aging and attentional demands on recovery from postural instability. Aging, 2, 155-161.

Stroop, J.R. (1935). Studies of interference in serial verbal reactions. Journal of Experimental Psychology, 18, 643661.

Sundermier, L., Woollacott, M., Roncesvalles, N., \& Jensen, J. (2001). The development of balance control in children: comparisons of EMG and kinetic variables and chronological and developmental groupings. Experimental Brain Research, 136, 340-350.

Swash, M., \& Fox, K.P. (1972). The effect of age on human skeletal muscle: studies of the morphology and innervation of muscle spindles. Journal of the Neurological Sciences, 16, 417-432.

Teasdale, N., Bard, C., LaRue, J., \& Fleury, M. (1993). On the cognitive penetrability of postural control. Experimental Aging Research, 19, 1-13.

Teasdale, N., \& Simoneau, M. (2001). Attentional demands for postural control: the effects of aging and sensory reintegration. Gait \& Posture, 14, 203-210.

Tucker, M.G., Kavanagh, J.J., Barrett, R.S., \& Morrison, S. (2008). Age-related differences in postural reaction time and coordination during voluntary sway movements. Human Movement Science, 27, 728-737.

Tucker, M.G., Kavanagh, J.J., Morrison, S., \& Barrett, R.S. (2009). Voluntary sway and rapid orthogonal transitions of voluntary sway in young adults, and low and high fall-risk older adults. Clinical Biomechanics, 24, 597-605.
Viel, S., Vaugoyeau, M., \& Assaiante, C. (2009). Adolescence: a transient period of proprioceptive neglect in sensory integration of postural control. Motor Control, 13, 25-42.

Vuillerme, N., \& Nafati, G. (2007). How attentional focus on body sway affects postural control during quiet standing? Psychological Research, 71, 192-200.

Vuillerme, N., \& Nougier, V. (2004). Attentional demand for regulating postural sway: the effect of expertise in gymnastics. Brain Research Bulletin, 63, 161-165.

Vuillerme, N., Nougier, V., \& Teasdale, N. (2000). Effects of a reaction time task on postural control in humans. Neuroscience Letters, 291, 77-80.

Wickens, C.D. (1974), Temporal limits of human information processing : a developmental study. Psychological Bulletin, 81, 739-755.

Wolff, D.R., Rose, J., Jones, V.K., Bloch, D.A., Oehlert, J.W., \& Gamble, J.G. (1998). Postural balance measurements for children and adolescents. Journal of Orthopaedic Research, 16, 271-275.

Woollacott, M., Shumway-Cook, A. (2002).Attention and the control of posture and gait: a review of an emerging area of research. Gait \&S Posture, 16, 1-14.

Wulf, G., \& Prinz, W. (2001). Directing attention to movement effects enhances learning: a review. Psychonomic Bulletin Review, 8, 648-660.

Yardley, L., Gardner, M., Bronstein, A., Davies, R., Buckwell, D., \& Luxon, L. (2001). Interference between postural control and mental task performance in patients with vestibular disorder and healthy controls. Journal of Neurology and Neurosurgery Psychiatry, 71, 48-52.

Yardley, L., Gardner, M., Leadbetter, A., \& Lavie, N. (1999). Effect of articulatory and mental tasks on postural control. NeuroReport, 10, 215-219.

Yogev, G., Giladi, N., Peretz, C., Springer, S., Simon, ES., \& Hausdorff, J.M. (2005). Dual tasking, gait rhythmicity, and Parkinson's disease: which aspects of gait are attention demanding? European Journal of Neuroscience, 22, $1248-1256$.

Zachry, T., Wulf, G., Mercer, J., \& Bezodis, N. (2005). Increased movement accuracy and reduced EMG activity as the result of adopting an external focus of attention. Brain Research Bulletin, 67, 304-309. 